\title{
Investigation on Electromagnetic Launching for Single Stage Coilgun
}

\author{
Hany M. Mohamed, Mahmoud A. Abdalla*, Abdelaziz A. Mitkees, and W. Sabry \\ Electromagnetic Wave Group, Electrical Engineering Branch. \\ MTC College University \\ Cairo, Egypt \\ *maaabdalla@ieee.org
}

\begin{abstract}
This paper presents the investigation on electromagnetic launch for single stage coilgun. The proposed coilgun and its excitation circuit were designed for launching small objects few meters. A transient simulation for the projectile motion through the launcher was done using transient finite element commercial software ANSYS MAXWELL. Also a comparative comparison between different parameters is introduced including the effect of number of turns, voltage, induced current and length of coil on the exit velocity of projectile while leaving the barrel. Detailed studied cases have been investigated. The results confirm a direct proportional relation between the velocity of projectile and number of turns, voltage and induced current in coil while confirming an inverse proportional relation with the length of coil.
\end{abstract}

Keywords-Electromagnetic launcher; Coilgun; Railgun; EM forces; Lorentzforce.

\section{INTRODUCTION}

Electromagnetic launcher technology (EML) is a strong candidate for launching projectiles with high velocities and to long distances. The launching speed may exceed some of conventional launchers. Railgun and Coilgun are the principle electromagnetic launchers which are being developed nowadays in researches. Both launchers convert very large amounts of electrical energy to mechanical energy (projectile kinetic energy) in very short time periods. The physical principles responsible for the driving force in each gun concept are different. As a result, they can be employed in different applications [1]. Electromagnetic (EM) Coilgun and EM Railgun have been demonstrated in laboratories. A lot of problems faced the fabrication of these launchers. Problems complicate the process of selecting an appropriate launcher and power supply system for a given application and technology level. However, each launcher and power supply system requirements have technological obstacles that prevent the system from being put in the field today.

Railgun research has been started in the 1970's at The Australian National University. The first tested railgun was $5 \mathrm{~m}$ long and used $1.6 \mathrm{MA}$ current to accelerate a $3 \mathrm{~g}$ projectile to $6 \mathrm{~km} / \mathrm{s}$. Later on, electromagnetic railgun launcher research has been done at various test facilities, including a lot of tests in army research laboratories in a lot of united state research institutes [2]. For examples, they have been used in for long-range shore bombardment, increasing ship-to-shore standoff distance, and improving ship survivability in combat situations [3], [4]. However, there are a lot of fundamental problems appeared in railgun research including compact pulsed power supplies, barrel and armature design considerations, integrated launch packages. A major challenge in designing railguns is the handling of its high currents, particularly at the armature rail interfaces [5].

Coilgun EML technology has been started in 1970's. They are mainly suitable for low profile projectile applications. In general coilgun has the advantage that the projectile need not physically contact the walls of the launch tube. If velocities needed for launching payloads is achieved it would be an important benefit due to eliminating a potential source of wear that would otherwise limit barrel life. A fundamental concern for coilguns is that the EM forces basically act in the radial direction in the launch tube, so that the projectile is squeezed in the radial direction to provide acceleration in the axial (launch) direction. Very high switching voltages are necessary to rapidly energize the drive coils while the projectile is on its way to the coil. 
The principal technical challenges for propulsive devices are achieving high thrust and high coil strength. Also it is required to achieve producing and controlling high power levels, and maintaining good weight, volume, and efficiency characteristics. Exciting of pulsed power and high voltage technology are found in the growing area of electromagnetic launch and propulsion. The force requirements range from a few Newtons to millions of Newtons, and the pulsed electrical power requirements range from kilowatts to gigawatt. For repetitive operation, the average prime power requirements range from a few watts to megawatts [6].

In this paper we introduce an investigation on electromagnetic launch for single stage coilgun; the employed coil is compact in length, wound around plastic tube of diameter $10 \mathrm{~mm}$. A transient simulation for the projectile motion through the launcher was done using transient finite element commercial software ANSYS MAXWELL. The employed coilgun was designed for low speed projectiles applications. The excitation circuit for the proposed coilgun is made by only five 1000UF capacitors charged at different values of voltage where in each case the projectile's velocity is measured. Also the effect of number of turns, induced current and length of coil will be introduced. Seven cases are studied for each parameter and its effect on the projectile's velocity is recorded as a function of time. Comparison figures for the effect of each selected parameter on the velocity of projectile are also introduced.

\section{THEORY}

A basic electromagnetic coilgun consists of a series of stator coils and an armature coil that moves along the stator coil axis. The coilgun may have an electrical terminal pair for the armature and each stator coil; this makes the analysis of coilgun be more complex [1]. The driving force in coilgun is the Lorentz Force. The coilgun structure forces exist on the stator and armature of coilgun are shown in Fig. 1. According to the right hand rule, the Lorentz Force imparts an axially directed accelerated force on the armature as shown in Fig.1. Coilgun operation involves the interaction of the current density, J (in the coil), and the quasi stationary magnetic field, $\mathrm{B}$, which are related by:

$$
J=\nabla \times B / \mu
$$

where $\mu$ is the permeability of space, equal to $4 \pi \times 10-7$ Henry/meter. As a result, the current is entirely azimuthal, that is circulating around the axis. Forces result from the current density crossed into the magnetic field:

$$
F=J \times B=\nabla \times B \times B / \mu
$$

In order for the magnetic field to produce a force, both it and its curl must be non zeros [7].

The magnetic field generated by the stator interacts with the armature current to produce axial force on the armature. As shown in (3), the axial force, that pushes the projectile, is given by the radial magnetic field times the current density. In other words, this force acts as a propulsive force.

$$
F_{a z}=J_{a \phi} \times-B_{s r}
$$

On the other hand, as a result of the stator and armature currents and their interacting with the magnetic field, radial force applied on both stator and armature, radial forces are generated in both of them.

The radial forces acted on both armature and stator are opposite to each other, where the force on armature is radially inside to the center of coil and the force acted on stator is radially outward. These forces are responsible for compressing the armature inside the stator making contactless between the stator and armature [1]. These forces can be expressed in (4), and (5), respectively.

$$
F_{s r}=-J_{s \phi} \times-B_{a z}
$$




$$
-F_{a r}=J_{a \phi} \times-B_{s z}
$$

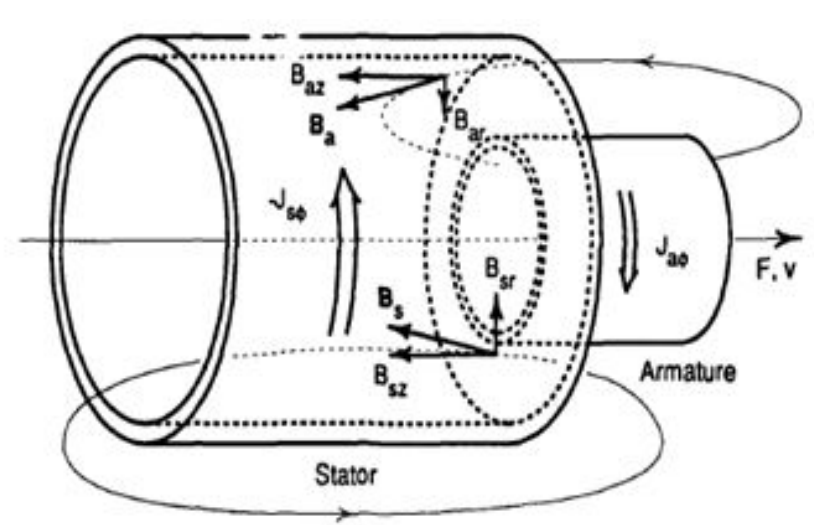

Fig. 1. Forces that exist on stator and armature coaxial coils inside a coilgun.

\section{SimULATION WORK}

The performance of the coilgun EM launching under the effect of the voltage stored in the capacitor bank is further investigated using transient finite element method simulation. The simulations investigate the projectile velocity and the induced current in coil as a function of time. The commercial software ANSYS MAXWELL is employed during simulation.

In our work, the effect of voltage, induced current in coil, coil's number of turns and coil's length will be investigated on an iron projectile which weighs $7.04 \mathrm{gm}$ and $21 \mathrm{~mm}$ in length and the exit velocity of projectile will be recorded. The relationship between each parameter and projectile's velocity will be shown. Seven different cases for each parameter will be investigated and total results will be shown in comparison figures.

\section{a- Effect of voltage and induced current in coil}

In this section we will discuss the effect of voltage and induced current passes through coil during discharging. We will focus our discussion on seven trials with different voltage values starting from 100 Volt to 400 Volt with 50 Volt difference between each trial and recording the output value of velocity for the projectile which moves along the barrel also getting the value of induced current passing through the coil in each voltage value. Two trials will be discussed in details and final figure for the seven trials will be shown. For the first trial the capacitor bank is charged up to 100 Volt and discharging process takes place through the coil to give maximum current pulse of $211.6 \mathrm{Amp}$ after $0.4 \mathrm{msec}$ from discharging as shown in figure 2 and $0.64 \mathrm{~m} / \mathrm{sec}$ exit velocity for the projectile as shown in figure 3. For the last trial a current pulse of 846.1 Amp can be detected when discharging a capacitor bank charged to 400 Volt as shown in figure 4; also the velocity of the projectile can be detected to be $25.2 \mathrm{~m} / \mathrm{sec}$ as shown in figure 5 .

Current pulses and projectile's velocity for the seven trials are shown in figures 6,7 simultaneously. It can be noticed that the effect of increasing the charged voltage on the two terminals of the capacitor bank will lead to an increase in the velocity of the projectile while moving inside the barrel; also an increase in the peak value of the discharging current pulse which make the magnetic field pushing the projectile more strengthen to get better velocity. We can compare between the voltage applied on the capacitor bank and the velocity of the projectile where it can be noticed the increase in velocity as charging voltage increases as shown in figure 8. Also the effect of current pulse on the velocity of projectile can be noticed in figure 9. These two figures can ensure an increase in velocity by $3837.5 \%$ and $299.86 \%$ increase in current pulse between first and last trials. 


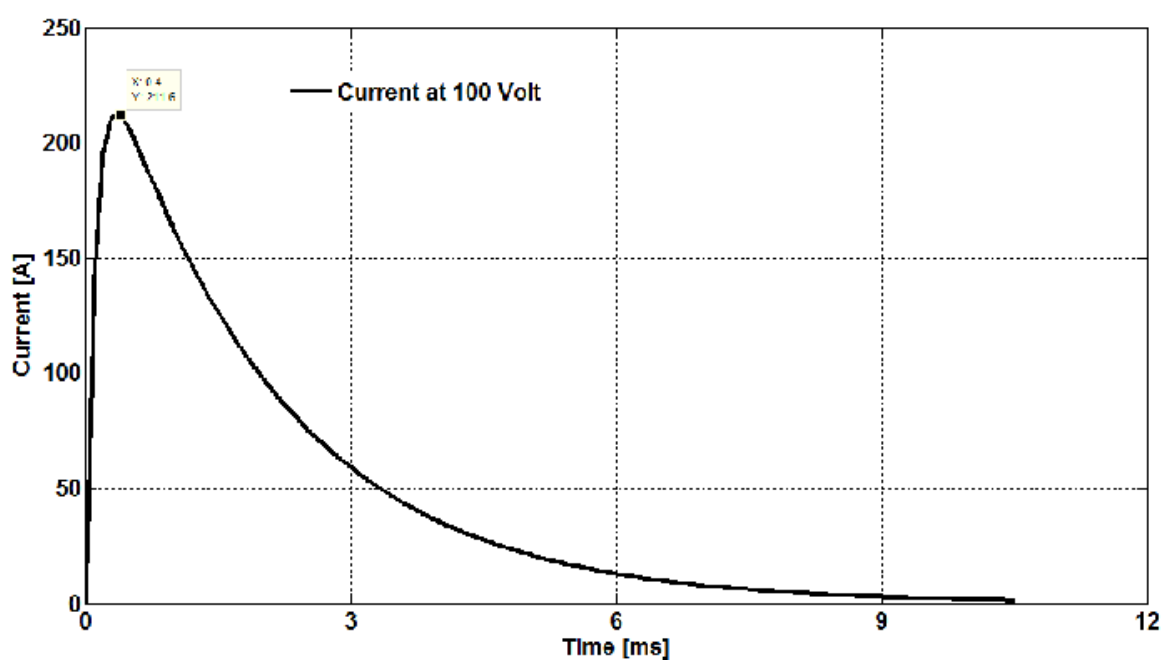

Fig. 2. Current pulse at 100 volt

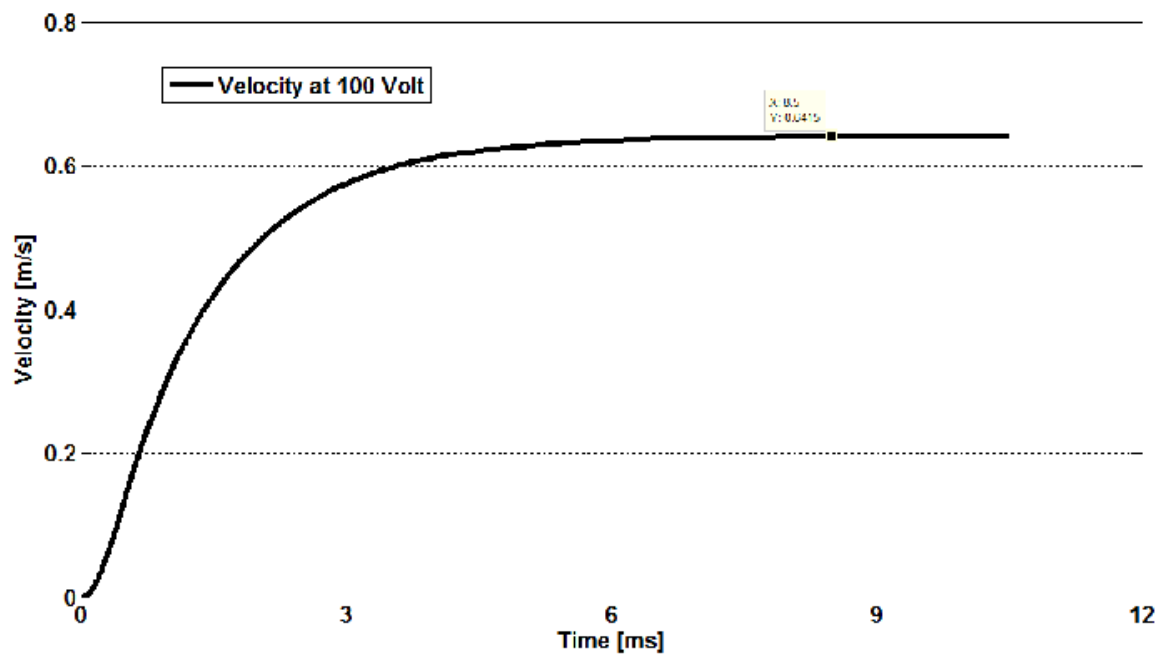

Fig. 3. Projectile's velocity during discharging

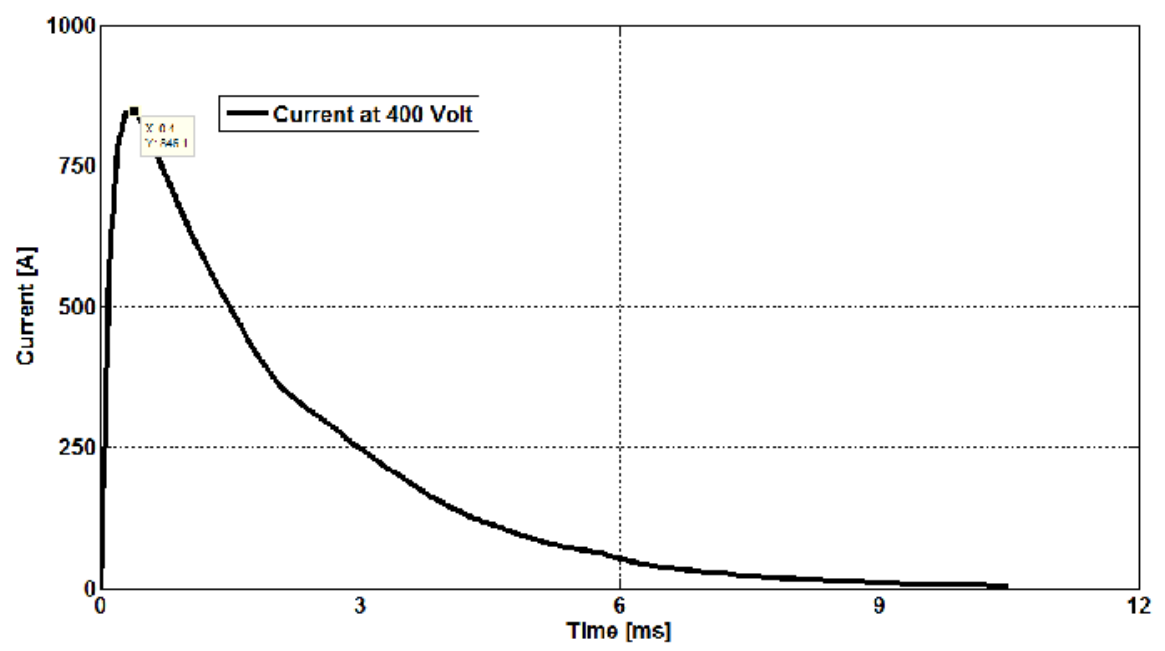

Fig. 4. Current pulse at 400 Volt 


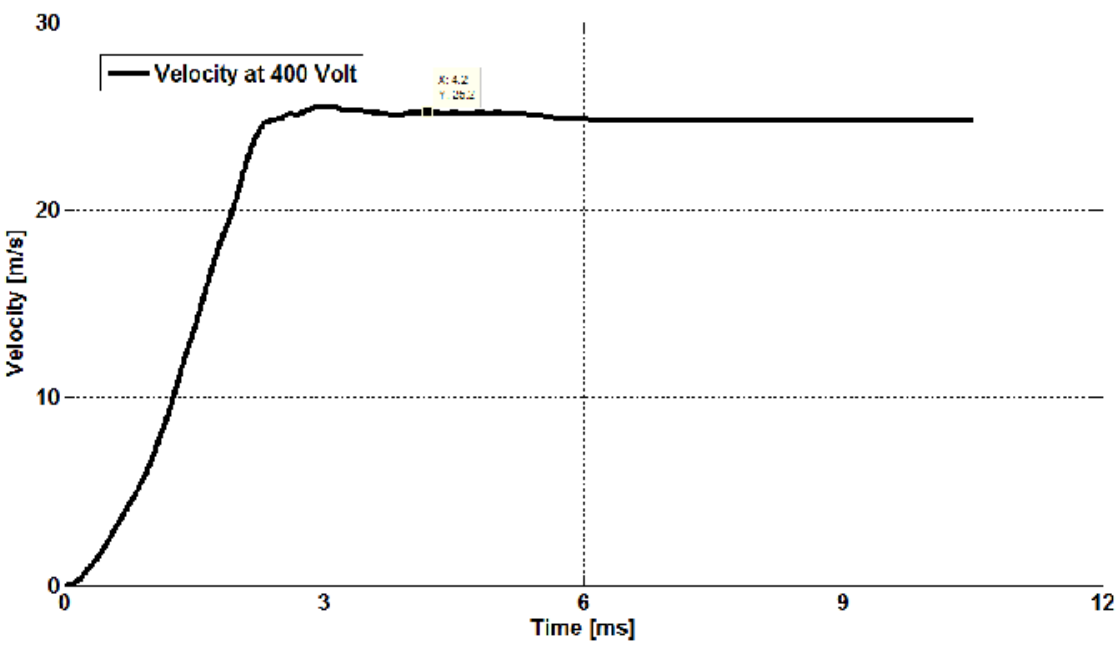

Fig. 5. Velocity of projectile during discharging

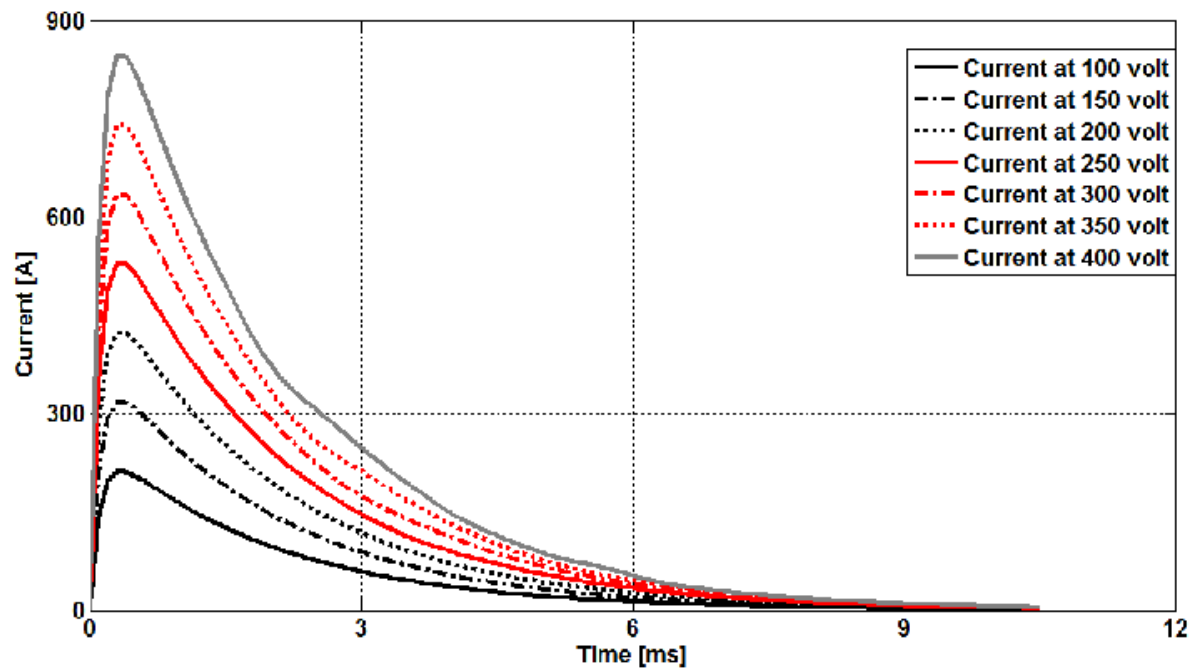

Fig. 6. Current pulses at different voltage

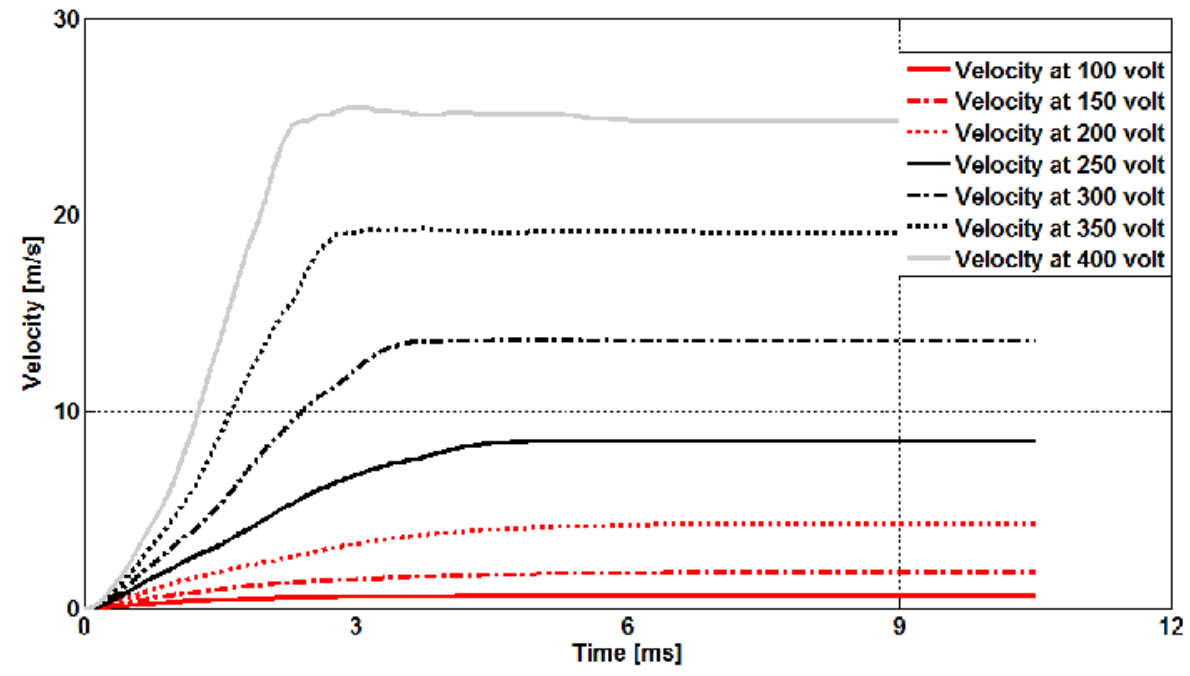

Fig. 7. Velocity of projectile at different voltage 


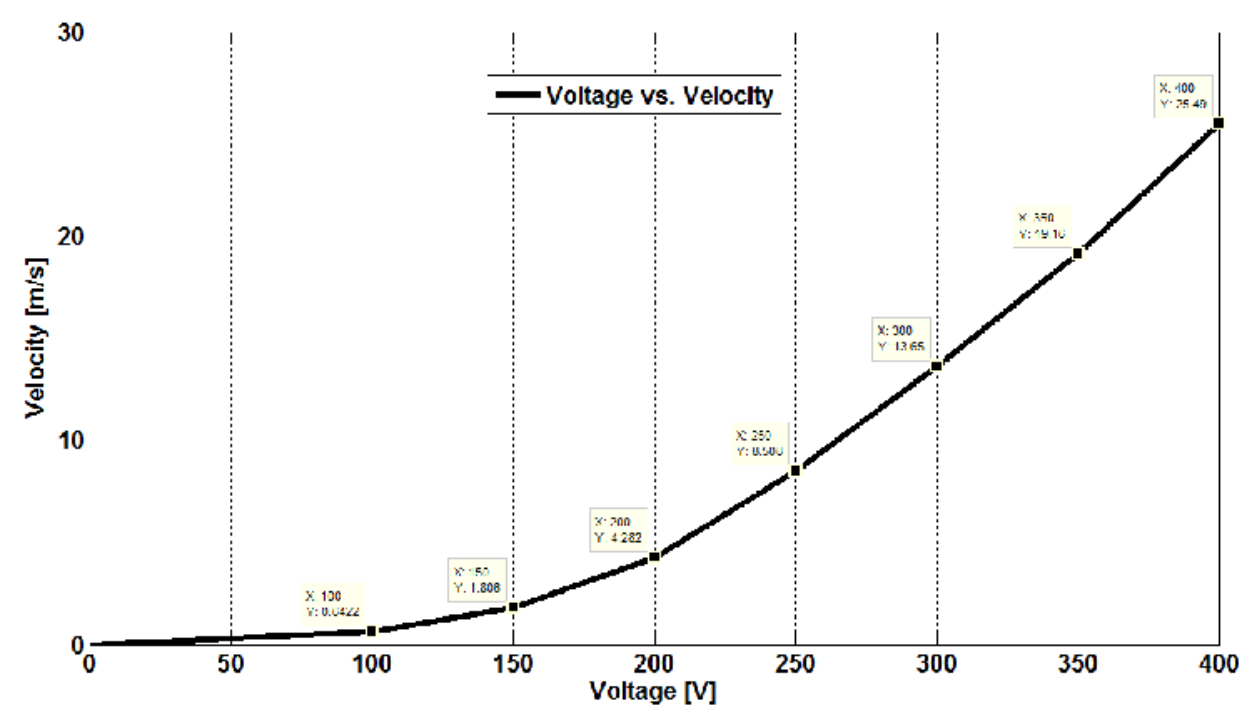

Fig. 8. Voltage vs. velocity

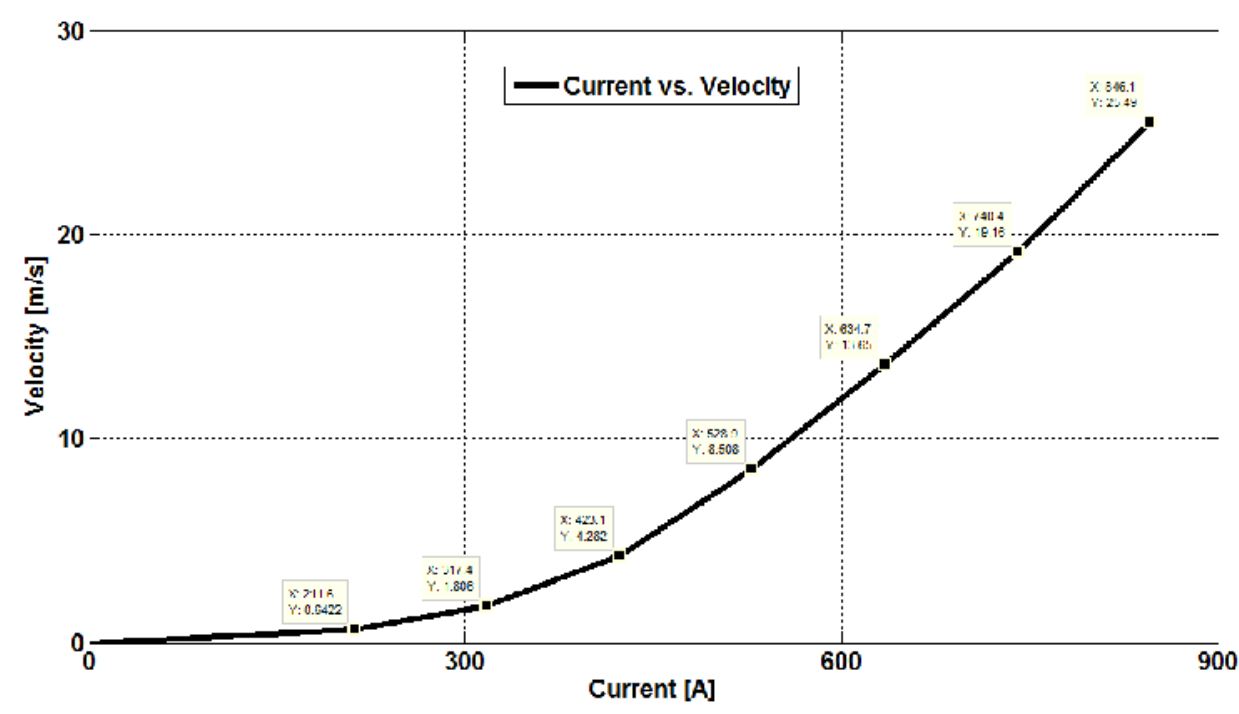

Fig. 9. Current vs. velocity

\section{b- Effect of number of turns}

In this section we will focus on the effect of changing the number of turns of coil winding on the velocity of projectile. As done before seven trials will be performed to show the effect of changing the number of turns on the velocity of projectile. These trials will vary from 50 turns to 200 turns with 25 turns increase in each trial. We confirm on keeping the length of the coil constant $(100 \mathrm{~mm})$ and the charge on the capacitor bank terminal unchanged (300 Volt). For the first trial; the number of turns is 50 turn which will deliver energy responsible for pushing the projectile to speed $0.6424 \mathrm{~m} / \mathrm{s}$. Increasing number of turns by 25 turn; the total number of turns in second trial will be 75 turn for the same length; this will provide an increase in projectile's velocity to be $1.825 \mathrm{~m} / \mathrm{s}$ with $184.1 \%$ increase than the first trial. Reaching to 100 turn for coil's number of turns will ensure the increase of projectile's velocity to reach $4.335 \mathrm{~m} / \mathrm{s}$; this will be $137.53 \%$ increase in velocity than second trial. By measuring the projectile's velocity when the number of turns is 125 turn it will be recorded to be $8.493 \mathrm{~m} / \mathrm{s}$; where it is more than the third trial by $95.92 \%$. Adding more turns for the coil to reach 150 turn will increase the velocity of the projectile to be $13.6 \mathrm{~m} / \mathrm{s}$ with $60.13 \%$ increase 
than the 125 turn coil. Achieving a velocity increase by $41.99 \%$ compared to the 150 turn coil when the number of turns reach 175 turn to record $19.31 \mathrm{~m} / \mathrm{s}$. Finally the 200 turn coil will provide us a projectile moving with velocity $25.23 \mathrm{~m} / \mathrm{s}$ providing $30.66 \%$ increase in velocity than the 175 turn coil trial. All these trials are recorded in figure 10; where a complete comparison for projectile's velocity with different number of turns is shown.

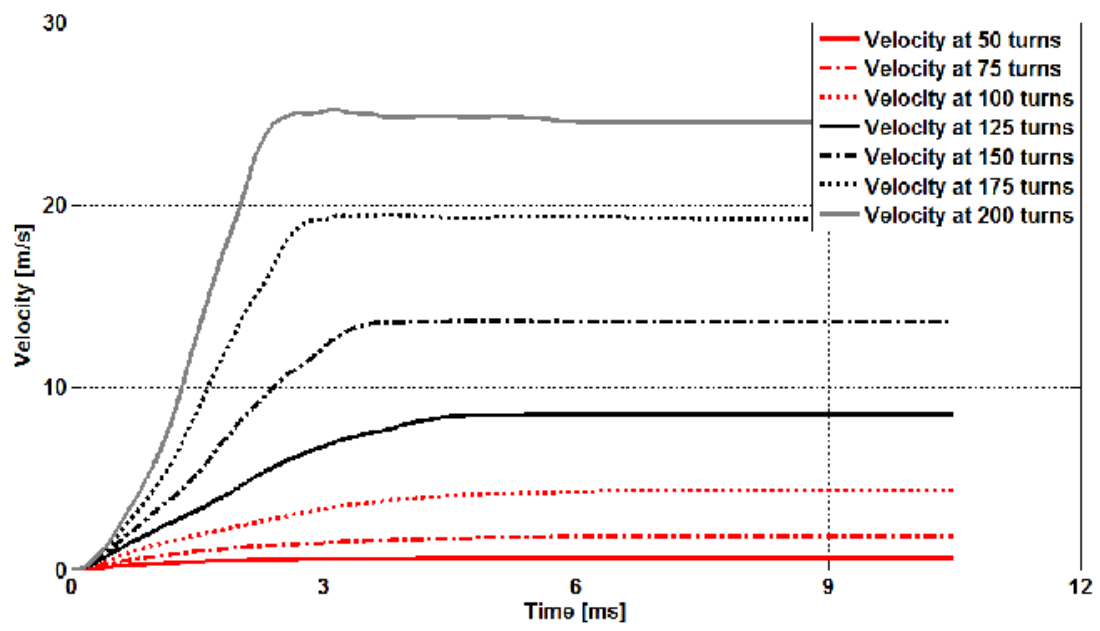

Fig. 10. Projectile's velocity at different number of turns

All these trials ensure that there is a direct proportional relation between the number of turns for coil and the projectile velocity; whereas the number of turns increases the projectile's velocity also increases and this is clearly shown in figure 11.

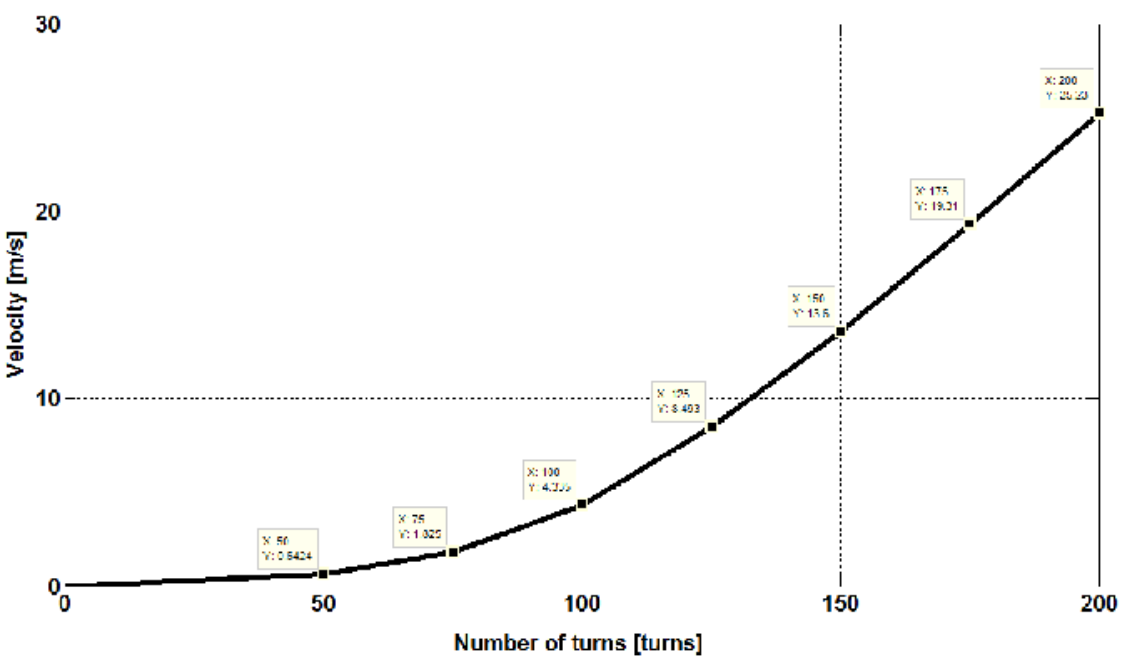

Fig. 11. Number of turns vs. velocity of projectile

\section{c- Effect of length of coil}

The Effect of length of coil on projectiles' velocity will be discussed in this section. Also seven different trials will be able to clarify the effect of length of coil on the velocity of projectile. First of all we will keep number of turns (100 turns) and applied voltage (300 Volt) on the terminals of the capacitor bank unchanged. The seven trials will begin with length $70 \mathrm{~mm}$ and ends with length $100 \mathrm{~mm}$ with $5 \mathrm{~mm}$ increase in each trial. For the first trial; the recorded velocity of projectile was $11.9 \mathrm{~m} / \mathrm{s}$ after discharging the capacitor bank. As we 
come to length of $100 \mathrm{~mm}$ for the coil; it is clear that the velocity of projectile has been decreased from 11.9 $\mathrm{m} / \mathrm{s}$ to $4.337 \mathrm{~m} / \mathrm{s}$ with $63.55 \%$ decrease from first trial. This may reflect the effect of length of coil which will be inversely proportional to the velocity of projectile which is oppositely unlike the effect of voltage and number of turns on the velocity of projectile. Figure 12 shows individually the values of projectile's velocity against each length of coil for each trial of the seventh trials. At last the effect of length of coil on projectile's velocity can be shown in figure 13 where as mentioned before as length of coil increases the velocity of projectile decreases while keeping number of turns and applied voltage on capacitor bank unchanged.

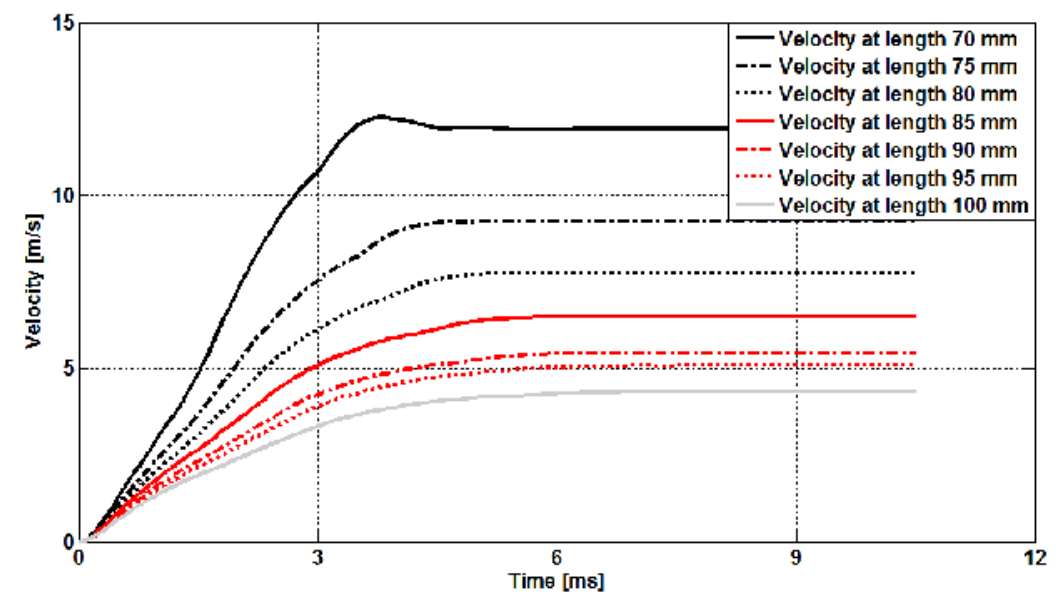

Fig. 12. Different Projectile's velocity at different coil length

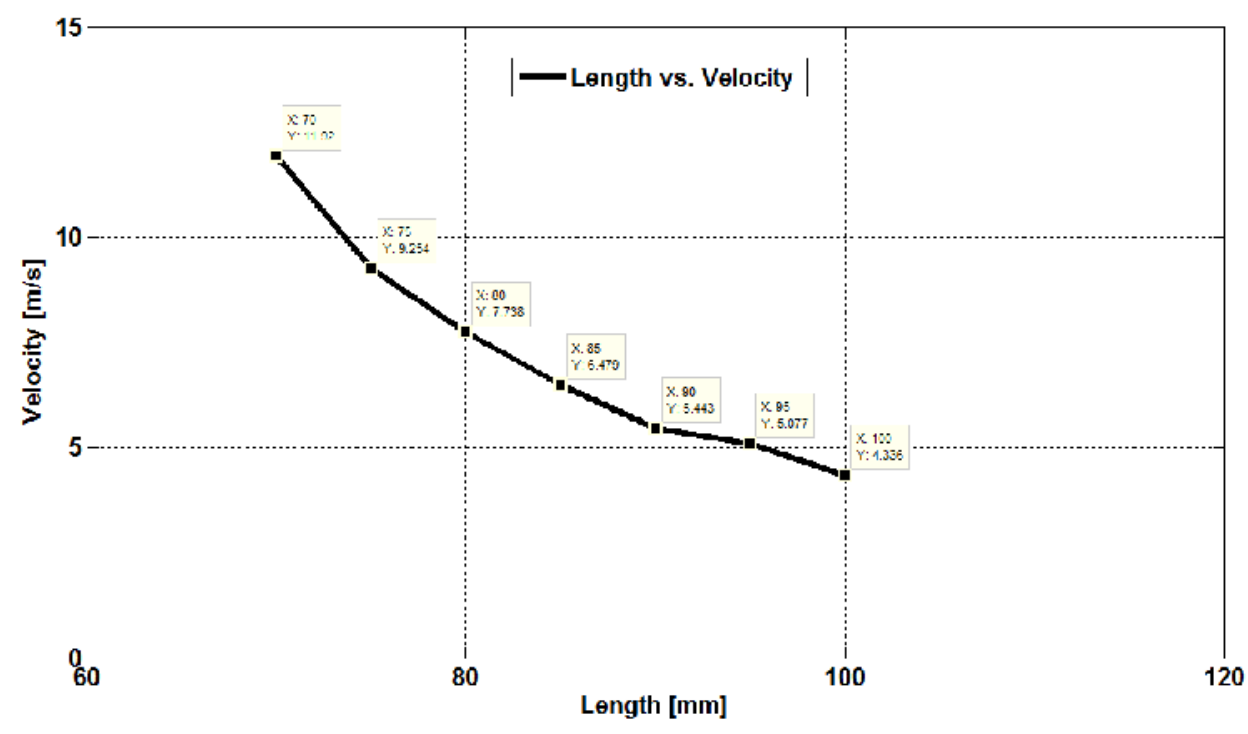

Fig. 13. Relation between length of coil and projectile's velocity

\section{CONCLUSION}

In this paper we have discussed investigation on electromagnetic launch for single stage coilgun. The effect of some parameters like voltage, induced current, number of turns of coil and coil's length on projectile's velocity is introduced using transient simulation program. The proposed simulation confirms the direct proportional relation between projectile's velocity and voltage, induced current and number of turns and confirms the inverse proportional relation between projectile's velocity and coil's length. 


\section{REFERENCES}

[1] Karl Edward Reinhard," A Methedology For Selecting An Electromagnetic Gun System”, The University of Texas at Austin, May, 1992.

[2] Ozkan Gurhan, "A Methedology To Measure The Metal Erosion On Recovered Armatures", Naval Postgraduate School, Monterey, California, December 2001.

[3] W.A. Walls, W.F. Weldon, S.B. Pratab, Palmer and D. Adams, "Applications of Electromagnetic guns to future naval platforms", IEEE Trans.Magn., pp.262-267, January 1999.

[4] Ian R.McNab, IEEE, Scott Fish, Francis Stefani, "Parameters for an Electromagnetic Naval Railgun", Institute for Advanced Technology, The University of Texas at Austin, 1999.

[5] A.Marshall, Richard, "Railgun Bore Geometry, round or square?”, IEEE Trans.Magn., Vol.35, Issue: 1, pp. 427 - 431, Jan 1999.

[6] Ronald J. Kaye, Bob N. Turman, and Steven L. Shope, “Applications Of Coilgun Electromagnetic Propulsion Technology”, IEEE, pp.703 - 707, June2002.

[7] Barry,M., “A Coilgun Design Primer”, IEEE Transactions On Magnetics. Vol 29. No. 1. January $1993 .$. 